\title{
SENSORES QUÍMICOS E FÍSICOS: UMA REVISÃO VOLTADA À ENGENHARIA BIOMÉDICA E SUAS APLICAÇÕES
}

\author{
CHEMICAL AND PHYSICAL SENSORS: A REVIEW FOCUSED ON \\ BIOMEDICAL ENGINEERING AND THEIR APPLICATIONS
}

\author{
Alícia Christmann Mattioni ${ }^{1}$, Pedro Machado Wurzel ${ }^{2}$ e \\ Paulo Jefferson Dias de Oliveira Evald ${ }^{3}$
}

\section{RESUMO}

Atualmente, os sensores biomédicos têm papel fundamental no auxílio de diagnósticos e tratamento de doenças. Estes sensores podem ser classificados como sensores físicos ou químicos, ambos com diversas aplicações. Neste contexto, os sensores físicos são utilizados para medir propriedades de natureza estritamente físicas. Já os sensores químicos, geralmente, são utilizados para realizar o reconhecimento de constituintes químicos e têm como um sub-grupo os biossensores, que são ferramentas de grande importância na área de pesquisa biomédica. Assim, este trabalho tem como objetivo realizar uma revisão dos recentes avanços de tais sensores, com ênfase nas aplicações biomédicas. Além disso, uma discussão sobre as tendências e perspectivas nesse campo é apresentada.

Palavras-chave: Instrumentação Clínica, Transdutores Biomédicos, Biossensores.

\begin{abstract}
Currently, biomedical sensors have a fundamental role in helping to diagnose and treat diseases. These sensors can be classified as physical or chemical sensors, both with different applications. In this context, physical sensors are used to measure properties of nature that are strictly physicals. On the other hand, chemical sensors, generally, are used to perform or recognize chemical components and have as a subgroup the biosensors, which are great important tools in the field of biomedical research. Thereby, this work has as main objective to review the recent advances of these sensors, with emphasis on biomedical applications. Besides, a discussion about tendencies and perspectives on this field is provided.
\end{abstract}

Keywords: Clinical Instrumentation, Biomedical Transducers, Biosensors.

\footnotetext{
${ }^{2}$ Discente do curso de Engenharia Biomédica da UFN. E-mail: alicia.mattioni@ufn.edu.br

${ }^{2}$ Discente do curso de Engenharia Biomédica da UFN. E-mail: pedro.wurzel@ufn.edu.br

${ }^{3}$ Professor Assistente da UFN. E-mail: paulo.evald@ufn.edu.br
} 


\section{INTRODUÇÃO}

A Engenharia Biomédica é a união da ciência e tecnologia que aplica princípios e métodos de engenharia para compreensão, definição e resolução de problemas em biologia e medicina. O principal objetivo dessa área da engenharia é desenvolver equipamentos e técnicas para o diagnóstico, a monitoramento e a terapia (DA CUNHA; FERLIN; PILLA JÚNIOR, 2004). Entre as grandes áreas de atuação do engenheiro biomédico, pode-se destacar a área de Instrumentação Biomédica, na qual há o estudo da eletrônica, de circuitos elétricos de potência, processamento analógico e digital de sinais, entre outros. Com este conhecimento, pode-se desenvolver soluções de hardwares e softwares para as aplicações biomédicas, como por exemplo os sensores.

Sensores servem para informar um circuito eletrônico a respeito de um evento que ocorre externamente sobre o qual ele deve atuar, ou a partir do qual ele deve comandar uma determinada ação (WENDLING, 2010). Para gerar um diagnóstico preciso de várias doenças, os profissionais precisam ter sinais biomédicos de alta qualidade gerados por diferentes órgãos humanos. Portanto, sensores biomédicos, como interface entre organismo humano e sistemas de computador, são muito importantes para adquirir informações médicas (LUO; ZHOU; LUO, 2016). Estes sensores podem ser classificados de diversas formas, mas em geral, se dividem em dois grandes grupos: sensores físicos e sensores químicos.

Sensores físicos são aqueles que medem propriedades de natureza estritamente físicas, como a massa, a viscosidade, o índice de refração, entre outras grandezas. Parâmetros como temperatura, fluxo, pressão, velocidade de agitação e densidade são largamente empregados nos mais diversos processos (YEO; LIM, 2016). De forma geral, sensores físicos são de fácil implementação, se comparados aos sensores químicos, com boas características metrológicas, e por conseguinte, apresentam baixos índices de inovação (TREVISAN; POPPI, 2006). Entretanto, em processos biomédicos, esses sensores são tão importantes quanto os sensores químicos.

Já os sensores químicos possuem dois tipos de extrema importância nas aplicações biomédicas: os eletrodos e sensores ópticos. O primeiro, engloba sensores de fenômenos elétricos no organismo e o segundo, utiliza-se da luz para coletar informações. Já nas aplicações dos sensores biomédicos, destacam-se os sensores eletroquímicos, sensores fotométricos e sensores bioanalíticos. Todos são utilizados para medir concentrações e monitorar atividades químicas no corpo. Além disso, os sensores químicos realizam o reconhecimento de constituintes químicos, como a concentração de determinada substância (TREVISAN; POPPI, 2006). Um sensor químico pode ser definido como um dispositivo que emprega um elemento de reconhecimento acoplado a um transdutor, onde o elemento de reconhecimento é o responsável pela interação seletiva com o analito de interesse. $\mathrm{O}$ transdutor tem como objetivo converter a energia do evento de reconhecimento em um sinal mensurável (TARLEY; SOTOMAYOR; KUBOTA, 2005). Portanto, sensores de identificação 
molecular baseados em processos bioquímicos (ou em reações) são convencionalmente chamados de biossensores, pois transcendem as definições usuais. Esses biossensores podem ser considerados como um sub-grupo dos sensores químicos (TREVISAN; POPPI, 2006).

Atualmente, os biossensores tem revolucionado diversas áreas (ROCCHITTA et al., 2016) e vem sendo usado como ferramentas de grande importância na área de pesquisa biomédica (ROSINI; D’ANTONA; POLLEGIONI, 2020). Ademais, eles são essenciais na saúde moderna, pois futuramente o seu desenvolvimento se tornará ainda mais fundamental, devido à grande demanda por medicamentos personalizados e ferramentas de diagnóstico de menor custo. Destaca-se que o reconhecimento seletivo por biossensores é realizado por biomoléculas (receptores biológicos), tais como anticorpos ou enzimas, que possuem a capacidade de suprir as demandas analíticas para uma ampla gama de analitos de interesse. No entanto, uma grande desvantagem destas biomoléculas em sensores está associada à pobre estabilidade química e física, resultando em uma falta de estabilidade operacional e de armazenagem, razões pelas quais os receptores artificiais têm atraído crescente interesse (TARLEY; SOTOMAYOR; KUBOTA, 2005).

Assim, é notável que os sensores são componentes fundamentais dos sistemas biomédicos tanto na identificação de patógenos e anomalias quanto no tratamento terapêutico do paciente. Devido a essa importância, essa área está em crescente expansão e continuará a ser continuamente desenvolvida em prol dos benefícios que propicia a humanidade. Portanto, este trabalho apresenta uma revisão das tecnologias sensoriais recentes com foco nas aplicações biomédicas, além de realizar uma discussão sobre as tendências e perspectivas dessa área.

\section{SENSORES FÍSICOS}

Atualmente, há uma vasta variedades de sensores físicos que têm sido bastante utilizados em diversas aplicações. Um primeiro exemplo são os sensores de fibra óptica, que estão sendo amplamente pesquisados (LIU et al., 2018) e que permitem uma medida direta de pressão, deformação e temperatura com uma boa precisão e estabilidade. Para tal, utilizam uma fonte de luz e um sistema de leitura, onde é possível realizar a medição de uma variedade de sinais multiplexados relacionados a deformação, vibração, temperatura e pressão ao longo de uma única fibra óptica (CUNHA, 2007). Na área biomédica, esses sensores podem ser aplicados na detecção de hiperemia ativa fisiológica funcional. No trabalho de Ripka e colaboradores (2014), tal instrumento foi instalado em uma bicicleta para coletar dados para fins de avaliação biomecânica e cinemática em praticantes de ciclismo. Durante os testes, a ferramenta mostrou-se útil e promissora na análise desses biossinais. Entretanto, ainda carece ser mais explorada e otimizada, para futuramente haver um barateamento da tecnologia envolvida nesses instrumentos (RIPKA et al., 2014).

Outro tipo de sensores físicos muito utilizados são os sensores de temperatura. Esses sensores, são utilizados para monitoração ou leitura imediata de temperatura. Em grande parte desses 
sensores, a medida de temperatura é realizada utilizando a propriedade física que relaciona o aumento da resistência elétrica ao aumento da temperatura, permitindo assim a leitura correspondente em escala definida dessa variação. Os sensores que se utilizam dessa propriedade são os chamados RTDs (Resistance Temperature Detectors) (MARTINAZZO; ORLANDO; URI, 2016). Esses sensores são amplamente utilizados na área médica, como por exemplo, em sensores de temperatura orais e nasais.

Ressalta-se ainda, outra grande aplicação de sensores físicos na área biomédica, que é no treinamento em biofeedback. Esse treinamento tem sido utilizado para o tratamento de diferentes quadros clínicos e para a prevenção/alívio de sintomas com relação ao estresse e ansiedade (LANTYER; VIANA; PADOVANI, 2013). Há diferentes tipos de instrumentos de biofeedback, como o biofeedback de variabilidade de frequência cardíaca (VFC), onde um sensor de pulso preso nos dedos é conectado a um computador e mostra o ritmo cardíaco em tempo real em um gráfico, revelando os níveis de ansiedade e estresse. Um outro instrumento de biofeedback comumente utilizado em associação à VFC, é o RSA (Respiratory Sinus Arhythmia), que mensura a arritmia sinusal respiratória, sendo capaz de medir a variação da frequência respiratória. Nesta aplicação, as alterações são informadas em tempo real por meio de um sensor digital infravermelho. Já o biofeedback eletroencefalográfico (EEG), ou neurofeedback, identifica os tipos de ondas cerebrais, a partir de sensores posicionados na cabeça do indivíduo. Essas ondas estão relacionadas aos estados de relaxamento e bem-estar, bem como está relacionada aos estados de meditação e hipnose.

Além dos biofeedbacks citados anteriormente, há ainda os biofeedbacks de respostas galvânicas da pele (GSR), que medem a resposta eletrodérmica por meio do posicionamento dos sensores nas pontas dos dedos indicador e médio da mão dominante do indivíduo. Esses dedos são estrategicamente selecionados, devido a condutividade que as extremidades possuem como indicadores emocionais. Por fim, mas não menos importante, há o biofeedback termal, que também permite o acoplamento de sensores nas extremidades das mãos ou dedos, sendo sensível à temperatura da pele por meio de pequenos vasos, pois em estados de maior relaxamento a temperatura tende a ser elevada (LANTYER; VIANA; PADOVANI, 2013).

A aplicação mais conhecida dos sensores físicos na área biomédica é no eletrocardiograma (ECG), onde a maior parte dos equipamentos de eletrocardiografia adquirem o sinal de forma não-invasiva, ou seja, por meio de eletrodos que não causam nenhum tipo de dor, risco de choques ou infecções (SCHWARZ, 2009). O eletrocardiograma (ECG) é o registro dos fenômenos elétricos que se originam durante a atividade cardíaca por meio de um aparelho chamado eletrocardiógrafo (RAMOS; SOUSA, 2007). O ECG pode ser adquirido pela medição da diferença de potencial de um eletrodo, posicionado em um ponto da pele, até um potencial de referência ou por meio da diferença de potencial elétrica entre dois eletrodos (SCHWARZ, 2009). Os eletrodos servem como auxiliares de extrema importância no diagnóstico de grande número de cardiopatias e outras condições (RAMOS; 
SOUSA, 2007). Antigamente, grandes dispositivos eram necessários para medição dessa diferença de potenciais. Já hoje em dia, há, comercialmente, pequenos ECGs com muita tecnologia embarcada, como por exemplo, comunicação wireless, além de auxiliarem na geração de diagnóstico de forma automatizada (SERHANI et al., 2020).

Em se tratando de aplicações biomecânicas, os sensores físicos também possuem grande relevância na área esportiva, onde cada vez mais há estudos dedicados a melhorar o desempenho dos atletas por meio de análises condicionais. Entretanto, o estudo da movimentação atlética humana requer análises específicas de acordo com cada esporte. Porém, de modo geral, para qualquer análise de movimento, é necessário a utilização de equipamentos que possibilitam a obtenção e análise dos dados gerados por atletas. Com isso, atualmente as unidades de sensores inerciais (Inertial Measurement Units - IMU) têm se tornado uma ferramenta importante para aquisição de dados relacionados ao movimento. Esses sensores são baratos, leves, pequenos, de fácil utilização e permitem a mobilidade quando integrados aos módulos de comunicação sem fio. Em suma, os sensores inerciais são caracterizados como sistemas de navegação inercial, ou seja, são capazes de medir movimentos lineares e/ou angulares pelo processamento de dados provenientes de acelerômetros e giroscópios (SANTOS; VIEIRA; STEVAN JR, 2016). Esses sensores são caracterizados por um conjunto de acelerômetros, giroscópios e magnetômetros. Com isso, eles são capazes de medir velocidade, orientação e força gravitacional, permitindo convenientemente seu uso em equipamentos médicos e esportivos.

Outro tipo de sensor físico que vem sendo bastante desenvolvido desde a última década são os chamados sensores vestíveis (KHAN et al., 2010). O uso desses sensores teve origem da necessidade de monitoramento de pacientes por grandes períodos de tempo (BONATO, 2003). Um exemplo de aplicação desses sensores foi mostrado por Jovanov e colaboradores (2005), onde implementaram uma rede de área corporal sem fio (WBAN - Wireless Body Area Network) vestível de sensores fisiológicos integrados a um sistema telemédico, permitindo a reabilitação de pacientes em sua residência, enquanto são supervisionados remotamente. O grande diferencial deste estudo, é que possibilita uma alternativa de reabilitação a distância, facilitando o acesso de tais serviços de saúde, por pacientes em áreas de difícil acesso. Embora seja muito útil e traga diversos benefícios aos pacientes, tal aplicação requer uma comunicação contínua e estável de internet (QI et al., 2018), o que ainda pode ser um desafio em localidades mais remotas. De qualquer forma, a integração de processadores e interfaces sem fio permitem o desenvolvimento de dispositivos de monitoramento inteligente em tamanhos reduzidos, mais leves (TRUNG; LEE, 2016), além de requer potência ultra baixa. Com essas vantagens, em relação a sistemas fixos de monitoramento, os sistemas de monitoração vestíveis vem ganhando cada vez mais atenção nos últimos tempos. A tendência em relação a esses sensores, é o uso de algoritmos inteligentes que aprendam a diagnosticar o paciente (QI et al., 2015), sendo um auxiliar poderoso ao médico. 


\section{SENSORES QUÍMICOS}

Assim como os sensores físicos, os sensores químicos apresentam uma grande usabilidade na área biomédica. Entre eles, destacam-se sensores eletroquímicos, sensores fotométricos e sensores bioanalíticos.

O desenvolvimento de sensores eletroquímicos é uma das áreas de maior e mais rápido crescimento dentro da Química Analítica, principalmente devido a uma crescente busca por sensores com melhores características, tais como alta sensibilidade, seletividade e estabilidade (FREIRE; PESSOA; KUBOTA, 2003). No ambiente celular, os dispositivos usualmente operam no modo amperométrico e apresentam menor seletividade. Os sensores amperométricos constituem-se em dispositivos mantidos em potencial fixo e que propiciam sinais de corrente faradaíca proporcionais à concentração do analito, em função de processos eletródicos que ocorrem na interface eletrodo/solução. Todavia, a corrente medida em experimentos eletroquímicos também possui um componente capacitivo, associado a mudanças da capacitância da dupla camada elétrica durante variações de potencial (LOWINSOHN; BERTOTTI, 2006).

Um sub-grupo dos sensores químicos que é recorrentemente utilizado em aplicações biomédicas são os biossensores. Os biossensores são dispositivos elétricos, ópticos, químicos ou mecânicos com capacidade de detectar espécies biológicas seletivamente (LEUNG; SHANKAR; MUTHARASAN, 2007). Eles são frequentemente modificados com entidade biológicas para aumentar sua seletividade e possuem amplas aplicações, incluindo detecção de biomarcadores para diagnóstico médico e detecção de patógenos e toxinas em certos alimentos e na água. Há estudos que revisam a utilização de biossensores de fibra óptica (FOBS - Fiber-optic biosensors), que são dispositivos que usam campo óptico para medir algumas espécies biológicas, como as células, proteínas e o DNA (Deoxyribonucleic Acid). Por possuir precisão e baixo custo, os FOBS são alternativas favoráveis aos métodos imunológicos tradicionais para medição das biomoléculas. Estes sensores usam as fibras ópticas como elemento de transdução e dependem exclusivamente de mecanismo de transdução óptica para detectar as biomoléculas alvo (LEUNG; SHANKAR; MUTHARASAN, 2007).

Há também muitos estudos que incorporam o uso da nanotecnologia nos biossensores. De acordo com alguns trabalhos da literatura, as análises em nanoescala têm sido estudadas e desenvolvidas para interações em nível celular e molecular em tempo real, fornecendo biossensores com sensibilidade muito alta quando comparados aos métodos convencionais (KOHLES et al., 2011). Dentre eles, nanopartículas, nanofios e nanotubos têm sido largamente aplicados em biodispositivos como nanomatrizes, nanobiossensores e sistemas microanalíticos (lab on a chip) (CHOI et al., 2007). Ressalta-se que os nanobiossensores são usados para detectar materiais químicos e biológicos. Assim, os sensores podem ser eletronicamente acoplados para responder à ligação de uma molécula individualmente (CALIL; SILVA, 2011).

Ainda associando o uso da nanotecnologia em biossensores, nos últimos anos tem sido amplamente desenvolvidos e utilizados nanotubos de carbono em biossensores, devido à sua alta sensibilidade, 
especificidade molecular, rapidez de análise, baixo custo e facilidade de utilização (REIS et al., 2018). Portanto, as nanoestruturas de carbono são materiais de imensa importância no estudo da nanociência por apresentarem características elétricas, físicas, mecânicas e químicas únicas, e são amplamente usadas para o desenvolvimento de equipamentos de alto desempenho (HU et al., 2010). Devido a essa elevada precisão, há diversos trabalhos que estudam a implementação desses biossensores com nanotubos de carbono na área clínica, principalmente para detecção de marcadores tumorais e de glucose (analitos associados a duas das doenças mais comuns no mundo, câncer e diabetes) (JUSTINO; ROCHA-SANTOS; DUARTE, 2013).

Por fim, outra classe de sensores químicos atualmente em constante exploração, são os sensores químicos e bioquímicos vestíveis. Com a detecção química e bioquímica vestível é possível fornecer novas informações fisiológicas não invasivas por meio da interação de vários fluidos corporais. Essas informações fornecem dados diagnósticos que permitem descobertas precoces de doenças, evitando seu desenvolvimento silencioso e melhorando assim a saúde dos pacientes (COYLE et al., 2014). Um exemplo de aplicação interessante nessa área é a utilização de dispositivos microfluidos baseados em papel ( $\mu$ PADs), que são integrados a um protetor bucal como sensor vestível para o monitoramento da glicose (CASTRO et al., 2019). Neste estudo, amostras de saliva humana foram coletadas de indivíduos saudáveis e de pacientes previamente diagnosticados com diabetes e os resultados obtidos foram satisfatórios, ou seja, evitando o agravamento da doença graças a detecção precoce de níveis nocivos de glicose.

\section{DISCUSSÃO}

Conforme observado nos trabalhos disponíveis na literatura, é indiscutível a importância dos sensores físicos e químicos nos dias de hoje. Na área biomédica, eles são instrumentos que facilitam estudos e permitem que diagnósticos e tratamentos sejam feitos de maneiras mais eficientes, rápidas e precisas. Porém há muito o que ser aprofundado neste âmbito.

Nos últimos anos, a pesquisa dedicada à área de sensores vestíveis evoluiu bastante, devido ao aumento de estudos e investimentos de empresas no desenvolvimento desses produtos para aplicações clínicas (BONATO, 2003). Como previamente apresentado, os sensores vestíveis vem sendo bastante utilizados, pois eles são capazes de fazer o diagnóstico, detecção bioquímica, fisiológica e dos movimentos. Além disso, tais sensores vem integrando tecnologias de comunicação sem fio a aplicativos de monitoramento remoto. Assim, tais redes de sensores auxiliam no diagnóstico e tratamento de usuários com doenças neurológicas, pulmonares e cardiovasculares (PATEL et al., 2012). Em vista disso, a aplicação desta tecnologia para o acompanhamento da saúde e bem estar dos indivíduos é um grande avanço e irá se desenvolver cada vez mais, integrando-se as interfaces populares como smartphones, por meio de aplicativos de interfaces simples e intuitivas, que podem inclusive, 
disparar alertas de emergência ao médico responsável em situações preventivas ou de mal súbito. Tal integração já está em seus primeiros passos com sensores físicos, e a tendência é que se expanda também aos sensores químicos.

Ressalta-se que os sistemas de monitoramento de saúde dos sensores vestíveis são compostos por vários sensores eletrônicos, atuadores, módulos de comunicação sem fio e unidades de processamento do sinal. Resumidamente, as medições que são obtidas pelos sensores conectados em uma rede sem fio de sensores corporais são transmitidos para um nó de processamento, que pode ser um smartphone ou até um computador (MAJUMDER; MONDAL; DEEN, 2017). Em Wang et al. (2015), os smartphones são aliados aos sensores vestíveis a favor da saúde e bem estar do paciente. Naquele trabalho, foi desenvolvido um serviço de mensagens curtas de texto para alertar sobre o nível de atividade física em adultos. O sistema foi testado em um grupo de adultos com sobrepeso e mostrou ser de grande utilidade. Ademais, hoje em dia com o uso dos sensores vestíveis já é possível constatar sintomas de depressão apenas monitorando parâmetros como quantidade de diálogos, intensidade de atividade física e da duração do sono usando pulseiras ou aplicativos de celular. Outro caso, a apneia do sono, que é uma das doenças que podem ser rapidamente ser detectada por meio do uso de um sensor vestível dedicado a medir a respiração, o ronco, tosses e o batimento cardíaco (FERRÃO, 2019). Pensando nessas novas aplicações que utilizam comunicação sem fio e smartphones, a tendência natural é o desenvolvimento de sistemas de cibersegurança dos dados gerados e da comunicação propriamente dita desses sistemas. Além disso, uma vez que os sensores dependem de baterias para seu correto funcionamento, a área de baterias de longa duração, como supercapacitores e de autoregeneração, transformando a energia absorvida como luz ou até pelo movimento em energia útil, são desafios que devem ser levados em consideração também para essa área.

Além dos sensores vestíveis, os sensores de microfibra, com a utilização de fibra óptica, também têm sido uma área de estudos recentes. Alguns trabalhos ressaltam a importância de sensores bioquímicos seletivos altamente sensíveis. Por exemplo, com a utilização de sensores de microfibra é possível realizar a detecção de diferentes sequências de DNA, proteínas, enzimas, ácidos nucléicos, anticorpos e diversos outras moléculas (LI et al., 2019). Esses dados têm fundamental importância em vários ramos, como no monitoramento de ambientes, controle de alimentos e diagnósticos médico.

Outra nova possibilidade na área de sensores físicos é o uso do grafeno. O grafeno é um material que tem atraído a atenção de muitos pesquisadores nos últimos anos. Ele tem sido utilizado como sensor óptico para a detecção de diversas amostras diferentes, como células, proteínas e pequenas moléculas (LI; ZHANG; XING, 2019). O grafeno apresenta as vantagens de ser barato, muito sensível a luz, pode atingir uma resposta ultrassensível e rápida. Recentemente, sensores de pressão capacitivos foram construídos usando materiais com permissividade dielétrica similar ao grafeno, o que aumentou significativamente o desempenho desses sensores (KUMAR et al., 2018). Portanto, a 
perspectiva futura de pesquisa de sensores físicos na área biomédica utilizando o grafeno é grande, devido as suas características favoráveis, aliadas ao seu baixo custo.

Já no âmbito dos sensores químicos, o grafeno também se mostra presente como uma alternativa viável. Em (HESS et al., 2011), os pesquisadores utilizaram um sensor de grafeno para detecção de atividade elétrica de células eletrogênicas. O grafeno é uma opção atraente para aplicações bioeletrônicas, por causa das suas grandes propriedades fisioquímicas, boa estabilidade química e biocompatibilidade, que são requisitos fundamentais para integração dos dispositivos aos sistemas biológicos. Ademais, a simples integração da eletrônica do grafeno com os substratos maleáveis, proporciona o desenvolvimento de dispositivos flexíveis, uma condição essencial para a elaboração de implantes biomédicos com redução de danos e cicatrizes nos tecido.

Além disso, sensores químicos implantáveis também são uma tendência na área de pesquisas em Engenharia Biomédica, pois eles têm a capacidade de fornecer dados confiáveis e em tempo real. Em (FROST; MEYERHOFF, 2002), foi mostrado o monitoramento simultâneo de várias medidas como pH sanguíneo, nível de eletrólitos, glicose, lactato e saturação do oxigênio. Esse monitoramento de múltiplas grandezas em tempo real têm uma importância clínica muito significativa, e é um dos maiores desafios na ciência analítica, sendo outro ponto que será continuamente investigado no futuro.

Outro elemento que apresenta novas possibilidades para o desenvolvimento de novos sensores é o silicone. O silicone é um material que permite a incorporação de diferentes sensores em um único equipamento. Isso propicia o seu uso para a criação de multisensores que podem ser grandes diferenciais na área biomédica, como no monitoramento da isquemia miocárdica. Em (ERRACHID et al., 2001), foi desenvolvida uma agulha multisensorial de silicone, capaz de detectar e medir íons como $\mathrm{K}+, \mathrm{H}+$ e a temperatura no tecido do miocárdio. Essa pesquisa continuou sendo realizada em experimentos in vivo, para futuramente usá-la durante cirurgias cardíacas. Posteriormente, novos trabalhos foram publicados e foi demonstrado que o dispositivo fabricado opera satisfatoriamente, tendo uma boa resposta a sensitividade e seletividade quando na solução existem cátions interferindo na medição (ZINE et al., 2003). Ressalta-se ainda que no campo médico, o estudo de organismos vivos é extremamente importante e necessário. Estudos com sondas fluorescentes de ácidos nucleicos mostram que elas podem ser usadas para estudos com células vivas, como para detecção celular, intracelular e comunicação entre células. Esse tipo de sonda tem se mostrado ótimo para o rastreamento de fenômenos moleculares complexos, e abre uma imensa gama de possibilidades para novas aplicações para estudos de organismos humanos, assim como de outros animais (WANG et al., 2013).

Para todas essas futuras perspectivas e tendências, é fundamental permanecer investindo na pesquisas e estudos de desenvolvimento de novas tecnologias, pois como discutido, elas são de grande relevância para humanidade, pois melhoram a qualidade de vida dos seus usuários. 


\section{CONCLUSÕES}

Neste trabalho foi mostrado a fundamental importância dos sensores físicos e químicos por meio da revisão de diversos trabalhos na área biomédica. Em particular, os sensores utilizados nas aplicações biomédicas são utilizados para a realização de diagnósticos, tratamentos e monitoração de pacientes. A partir dessa revisão, foi possível apontar as tendências tecnológicas nos sistemas de sensoriamento biomédicos, tais como: sensores vestíveis, comunicação e transmissão de dados sem fio, uso de aplicativos de monitoração e alerta da condição de saúde do paciente, além da expansão de aplicações biomédicas que utilizam smartphones como interface de comunicação e monitoramento. Além disso, também notou-se o crescente uso de alguns materiais como silicone e grafeno, devido as suas características fisioquímicas e baixo custo. Ademais, pesquisas com microfibra, rede de sensores integrados e biossensores estão em contínua expansão. Naturalmente, com o passar dos anos, a demanda do mercado por inovações tecnológicas tem crescido bastante. Para fornecer essas tecnologias, a multidisciplinaridade é de extrema relevância, onde a Química, a Física, a Biologia e a Engenharia caminham juntas para achar soluções eficazes e, sempre que possível, simples e de baixo custo. Hoje em dia, aliado aos conhecimentos já mencionados, é integrado a Computação e a Ciência de Dados, fornecendo novas possibilidades e desafios no desenvolvimento de novas soluções em favor da saúde e bem estar dos pacientes.

\section{REFERÊNCIAS}

BONATO, P. Wearable Sensors/Systems and Their Impact on Biomedical Engineering. IEEE Engineering in Medicine and Biology Magazine, v. 22, n. 3, p. 18-20, 2003. Disponível em: https://bit.ly/ 3g6sDIz

CALIL, S. S.; SILVA, P. d. Biossensores: estrutura, funcionamento e aplicabilidade. Instituto de Estudos Farmacêutico, 2011. Disponível em: https://bit.ly/2I6QyuV

CASTRO, L. F.; DE FREITAS, S. V.; DUARTE, L. C.; DE SOUZA, J. A. C.; PAIXÃO, T. R. L.C.; COLTRO, W. K. T. Salivary diagnostics on paper microfluidic devices and their use as wearable sensors for glucose monitoring. Analytical and Bioanalytical Chemistry, v . 411, n. 1, p. 4919-4928, 2019. Disponível em: https://bit.ly/3oeWT6I

CHOI, J.; OH, B.; JIM, Y.; MIN, J. Nanotechnology in biodevices. Journal of Microbiology and Biotechnology, v. 17, n. 1, p. 5-14, 2007. Disponível em: https://bit.ly/3ooaVTB 
COYLE, S.; CURTO, V. F.; BENITO-LOPEZ, F.; FLOREA, L.; DIAMOND, D. Wearable Bio and

Chemical Sensors. Wearable Sensors: Fundamentals, Implementation and Applications, p. 65-83, 2014. Disponível em: https://bit.ly/33CR4bm

CUNHA, J. R. F. A. da. Modelo teórico de sensores ópticos baseados em fibras com grade de bragg. Dissertação (Mestrado em Física), Universidade Federal do Pará (UFPA), 2007. Disponível em: https://bit.ly/33Hhp8t

DA CUNHA, J. C., FERLIN, E. P. , PILlA JÚNIOR, V. O Desenvolvimento de Pesquisas em Engenharia Biomédica no Nível de Graduação no UnicenP. WCETE 2004 - World Congress on Engineering and Technology Education, p. 1-4, 2004. Disponível em: https://bit.ly/36AJ3G7

ERRACHID, A.; IVORRA, A.; AGUILÓ, J.; VILLA, R.; ZINE, N.; BAUSELLS, J.. New technology for multi-sensor silicon needles for biomedical applications. Sensors and Actuators B. v. 78, n. 3, p. 279-284. 2001. Disponível em: https://bit.ly/39F8tUW

FERRÃO, R. Wearables: Dispositivos inteligentes para saúde e bem-estar. Universidade Virtual do Estado de São Paulo - UNIVESP, 2019. Disponível em: https://bit.ly/33EH1Bw

FREIRE, R. S.; PESSOA, C. A.; KUBOTA, L. T. Emprego de monocamadas auto-organizadas no desenvolvimento de sensores eletroquímicos. Química Nova, SciELO Brasil, v. 26, n. 3, p. 381-389, 2003. Disponível em: https://bit.ly/3gbm07N

FROST, M. C.; MEYERHOFF, M. E. Implantable chemical sensors for real-time clinical monitoring: progress and challenges. Current Opinion in Chemical Biology, v. 6, n. 5, p. 633-641, 2002. Disponível em: https://bit.ly/37vXUAL

HESS, L. H.; JANSEN, M.; MABECK, V.; HAUF, M. V.; SEIFERT, M.; STUTZMANN, M. SHARP, I. D.; OFFENHAUSSER, A.; ARRIDO, J. A. Graphene Transistor Arrays for Recording Action Potentials from Electrogenic Cells. Advanced Materials, v. 23, n. 43, p. 5045- 5049, 2011. Disponível em: https://bit.ly/2L4A33C

HU, P. , ZHANG, J.; LE L.; ZHENLONG, W.; O’NEILL, W.; ESTRELA, P. Carbon nanostructure-based field-effect transistors for label-free chemical/biological sensors. Sensors, v. 10, n. 5, p. 51335159, 2010. Disponível em: https://bit.ly/2JFhDFR 
JOVANOV, E.; MILENKOVIC, A.; OTTO, C.; DE GROEN, P. C. A wireless body area network of intelligent motion sensors for computer assisted physical rehabilitation. Journal of NeuroEngineering and Rehabilitation, v. 2, n. 6, p. 1-10, 2005. Disponível em: https://bit.ly/39A09Wt6

JUSTINO, C. I.; ROCHA-SANTOS, T. A.; DUARTE, A. C. Biossensores com nanotubos de carbono para aplicações clínicas: avanços tecnológicos. Revista Captar: Ciência e Ambiente para Todos, v. 4, n. 1, p. 1-12, 2013. Disponível em: https://bit.ly/2JFi3vV

KHAN, A. M.; LEE, Y. K.; LEE, S. Y.; KIM, T. S. A triaxial accelerometer-based physical-activity recognition via augmented-signal features and a hierarchical recognizer. IEEE Transactions on Information Technology in Biomedicine, v. 14, n. 5, p. 1166-1172, 2010. Disponível em: https://bit.ly/ 2L4ApY0

KOHLES, S. S.; BRADSHAW, S.; MASON, S. S.; LOOFT, F. A multivariate logistical model for identifying the compressive sensitivity of single rat tactile receptors as nanobiosensors. Journal of nanotechnology in engineering and medicine, American Society of Mechanical Engineers Digital Collection, v. 2, n. 1, p. 1-14, 2011. Disponível em: https://bit.ly/3g4AaaF

KUMAR, R., SINGH, R.; HUI, D.; FEO, L.; FRATERNALI, F. Graphene as biomedical sensing element: State of art review and potential engineering applications. Composites Part B 134, p. 193-206. 2018. Disponível em: https://bit.ly/2JH7cl8

LANTYER, A. d. S.; VIANA, M. d. B.; PADOVANI, R. d. C. Biofeedback en el tratamiento de transtornos relacionados con el estrés y la ansiedad: una revisión crítica. Psico-USF, SciELO Brasil, v. 18, n. 1, p. 131-140, 2013. Disponível em: https://bit.ly/2JI2LXq

LEUNG, A.; SHANKAR, P. M.; MUTHARASAN, R. A review of fiber-optic biosensors. Sensors and Actuators B: Chemical, v. 125, n. 2, p. 688-703, 2007. Disponível em: https://bit.ly/3onOMoL

LI, Z; ZHANG, W; XING, F. Graphene Optical Biosensors. International Journal of Molecular Sciences. v. 20, n. 10, 2461-2479, 2019. Disponível em: https://bit.ly/3ol0BvL

LI, Y., XU, Z.; TAN, S.; FANG, F.; YANG, L.; YUAN, B.; SUN, Q. Recent advances in microfiber sensors for highly sensitive biochemical detection. Journal of Physics D: Applied Physics, v. 52 , n. 49, p. 493002, 2019. Disponível em: https://bit.ly/3mDr1Z4 
LIU, I. C.; CHEN, P. C.; CHAU, L. K.; CHANG, G. E. Optofluidic refractive-index sensors employing bent waveguide structures for low-cost, rapid chemical and biomedical sensing. Optics express, v. 26, n. 1, p. 273-283, 2018. Disponível em: https://bit.ly/2JuxUO4

LOWINSOHN, D.; BERTOTTI, M. Sensores eletroquímicos: considerações sobre mecanismos de funcionamento e aplicações no monitoramento de espécies químicas em ambientes microscópicos. Química Nova, SciELO Brasil, v. 29, n. 6, p. 1318-1325, 2006. Disponível em: https://bit.ly/2JFiyWP

LUO, Y.; ZHOU, Q.; LUO, W. A brief review of biomedical sensors and robotics sensors. American Journal of Engineering Research, v. 5, n. 3, p. 191-194, 2016. Disponível em: https://bit.ly/31EI8se

MAJUMDER, S.; MONDAL, T.; DEEN, J. Wearable Sensors for Remote Health Monitoring. Sensors, v. 130, n; 17. p. 1-16, 2017. Disponível em: https://bit.ly/37AaXBn

MARTINAZZO, C. A.; ORLANDO, T.; URI, E. da. Comparação entre três tipos e sensores de temperatura em associação com arduíno. PERSPECTIVA, Erechim, v. 40, n. 151, p. 93-104, 2016. Disponível em: https://bit.ly/3g4ARkh

PATEL, S.; PARK, H.; BONATO, P. ; CHAN, L.; RODGERS, M. M. A review of wearable sensors and systems with application in rehabilitation. Journal of NeuroEngineering and Rehabilitation, v. 21, n. 9, p. 1-17, 2012. Disponível em: https://bit.ly/37qI1vq

QI, J.; YANG, P. ; FAN, D.; DENG, Z. A survey of physical activity monitoring and assessment using internet of things technology. In: 2015 IEEE International Conference on Computer and Information Technology; Ubiquitous Computing and Communications; Dependable, Autonomic and Secure Computing; Pervasive Intelligence and Computing. IEEE, p. 2353-2358. 2015; Disponível em: https://bit.ly/3mCatAU

QI, J.; YANG, P. ; WARAICH, A.; DENG, Z.; ZHAO, Y.; YANG, Y. Examining sensor-based physical activity recognition and monitoring for healthcare using Internet of Things: A systematic review. Journal of biomedical informatics, v. 87, p. 138-153, 2018. Disponível em: https://bit.ly/37vhu02

RAMOS, Â. P. ; SOUSA, B. S. Eletrocardiograma: princípios, conceitos e aplicações. Centro de Estudos de Fisiologia do Exercício, 2007. Disponível em: https://bit.ly/39BsUSr 
REIS, S. P. ; GLÓRIA, J. C.; ALMEIDA, M. E. M.; ARAUJO, F.; ALVES, K. C. A.; PEREIRA, K. D. E. S.; MANZATO, L.; MARIÚBA, L. A. M. Nanotubos de carbono: conceitos gerais e aplicação em biosenssores. Scientia Amazonia, v. 7, n. 1, p. 53-59, 2018. Disponível em: https://bit.ly/3qqo2FF

RIPKA, W.; DUTRA, G.; ULBRITCH, L.; MARTELLI, C.; GEWEHR, P. M. Sensor de fibra óptica para deteç̧ão de hiperemia ativa fisiológica funcional em teste ergométrico. In: XXIV Congresso Brasileiro de Engenharia Biomédica (CBEB), 2014. p. 1-4. Disponível em: https://bit.ly/31Dc08i

ROCCHITTA, G.; SPANU, A.; BABUDIERI, S.; LATTE, G.; MADEDDU, G.; GALLERI, G.; GALLERI, G.; NUVOLI, S.; BAGELLA, P.; DEMARTIS, M. I.; FIORE, V.; MANETTI, R.; SERRA, P. A. Enzyme biosensors for biomedical applications: Strategies for safeguarding analytical performances in biological fluids. Sensors, v. 16, n. 6, p. 780, 2016. Disponível em: https://bit.ly/39CvqYF

ROSINI, E.; D’ANTONA, P. ; POLLEGIONI, L. Biosensors for D-Amino Acids: Detection Methods and Applications. International Journal of Molecular Sciences, v. 21, n. 13, p. 4574, 2020. Disponível em: https://bit.ly/2JA2QfT

SANTOS, C. P; M. E. M. VIEIRA; STEVAN JR, S. L. Sensores inerciais aplicados à marcha humana no esporte. In: Seminário de Eletrônica e Automação (SEA), 2016. p. 1-6. Disponível em: https://bit.ly/33F5jwG

SCHWARZ, L. Artigo de revisão: eletrocardiograma. Revista Ilha Digital, v. 1, p. 3-19, 2009. Disponível em: https://bit.ly/37LxoDN

SERHANI, M. A., T EL KASSABI, H., ISMAIL, H., \& NUJUM NAVAZ, A.. ECG Monitoring Systems: Review, Architecture, Processes, and Key Challenges. Sensors, v. 20, n. 6, p. 1796, 2020. Disponível em: https://bit.ly/36A5hYu

TARLEY, C. R. T.; SOTOMAYOR, M. D. P. T.; KUBOTA, L. T. Polímeros biomiméticos em química analítica. parte 2: aplicações de mip ("molecularly imprinted polymers") no desenvolvimento de sensores químicos. Química Nova, SciELO Brasil, v. 28, n. 6, p. 1087-1101, 2005. Disponível em: https://bit.ly/33FI41Z

TREVISAN, M. G.; POPPI, R. J. Química analítica de processos. Química Nova, v. 29, n. 5, p. 10651071, 2006. Disponível em: https://bit.ly/36ybsfU 
TRUNG, T. Q.; LEE, N. Flexible and stretchable physical sensor integrated platforms for wearable human-activity monitoringand personal healthcare. Advanced materials, v. 28, n. 22, p. 4338-4372, 2016. Disponível em: https://bit.ly/2JIS5aM

WANG, J. B.; CADMUS-BERTRAM, L. A.; NATARAJAN, L.; WHITE, M. M.; MADANAT, H.; NICHOLS, J. F.; PIERCE, J. P. Wearable Sensor/Device (Fitbit One) and SMS Text-Messaging Prompts to Increase Physical Activity in Overweight and Obese Adults: A Randomized Controlled Trial. TELEMEDICINE and e-HEALTH, v. 25, n. 10, p. 782-792, 2015. Disponível em: https://bit.ly/2JoadY1

WANG, K.; CADMUS-BERTRAM, L. A.; NATARAJAN, L.; WHITE, M.; MADANAT, H.; NICHOLS, J.; AYALA, G.; PIERCE, J. Recent advances in fluorescent nucleic acid probes for living cell studies. Analyst, v. 138, n. 1, p. 62-71, 2013. Disponível em: https://rsc.li/2Iaq9fM

WENDLING, M. Sensores. Universidade Estadual Paulista. São Paulo, v.1, p. 1-20, 2010. Disponível em: https://bit.ly/33FhObq

YEO, J. C.; LIM, C. T. Emerging flexible and wearable physical sensing platforms for healthcare and biomedical applications. Microsystems \& Nanoengineering, v. 2, n. 1, p. 1-19, 2016. Disponível em: https://go.nature.com/36yQbTa

ZINE, N., BAUSSELS, J., IVORRA, A., AGUILLO, J., ZABALA, M., TEIXIDOR, F. MASALLES, C. VIÑAS C., ERRACHID, A. Hydrogen-selective microelectrodes based on silicon needles. Sensors and Actuators B: Chemical, v. 91, p. 76-82, 2003. Disponível em: https://bit.ly/3mCnPgu 
